\title{
Comparison of causative microorganisms of posttraumatic endophthalmitis with and without retained intraocular foreign bodies
}

\author{
Yao Yang, Feng Mei, Jiaqi Lin, Jingyu Liao, Kaili Wu ${ }^{*}$ and Fang Duan ${ }^{*}$
}

\begin{abstract}
Background: The goals of this work were to report the demographic characteristics of patients with clinically diagnosed endophthalmitis with or without intraocular foreign bodies (IOFBs) and to analyze the causative microorganisms.

Methods: A retrospective analysis was conducted on 1257 patients with clinically diagnosed posttraumatic endophthalmitis who were admitted to Zhongshan Ophthalmic Center between January 1, 2013, and August 31, 2020.

Results: Of the 1257 patients with clinically diagnosed posttraumatic endophthalmitis, 452 (36.0\%) patients had IOFBs. Male dominance was more common among the patients with IOFBs than the patients without IOFBs. The average age of the patients with IOFBs was older than that of the patients without IOFBs. The most common microbial pathogens in these two groups were Gram-positive cocci and Gram-negative bacilli. Gram-positive bacilli were more common in the patients with IOFBs than in those without IOFBs (17.9 vs. 9.4\%), and Bacillus spp. accounted for 12.6 and 5.5\%, respectively. Fungi were less abundant in the patients with IOFBs than in those without IOFBs (8.0 vs. 15.6\%).

Conclusions: Patients with IOFBs were mostly male and older than those without IOFBs. Gram-positive bacilli were more common and fungi were less common in patients with IOFBs than in those without IOFBs.
\end{abstract}

Keywords: Open globe injury, Endophthalmitis, Intraocular foreign body, Pathogens

\section{Background}

Posttraumatic endophthalmitis is a potentially devastating complication of open globe injuries and is of particular concern due to its tendency toward rapid vision loss and even blindness. Retained intraocular foreign bodies (IOFBs) occur in $18-41 \%$ of ocular trauma cases [1] and escalate the development of endophthalmitis. The prevalence of posttraumatic endophthalmitis has been reported to vary widely, i.e., from 0.9 to $11.9 \%$ [2-5], and

*Correspondence: wukaili@mail.sysu.edu.cn; duanfangg@126.com Zhongshan Ophthalmic Center, State Key Laboratory of Ophthalmology, Sun Yat-sen University, 54 Xianlie Road, Guangzhou 510060, China may be much higher, i.e., from 6.9 to $30 \%$, in cases of intraocular foreign body (IOFB) injury [6-8]. In addition, IOFBs are reported to be present in $43 \%$ of eyes diagnosed with traumatic endophthalmitis [9].

The spectrum of causative organisms of posttraumatic endophthalmitis varies in different regions. Most of the previous studies have reported that bacteria account for approximately $80-90 \%$ of culture-positive cases, and Gram-positive cocci are the most common isolates, followed by Gram-negative bacilli and Gram-positive bacilli $[10,11]$. IOFBs have been reported as risk factors in many studies. Several studies have reported high incidence of Bacillus species endophthalmitis following open globe original author(s) and the source, provide a link to the Creative Commons licence, and indicate if changes were made. The images or other third party material in this article are included in the article's Creative Commons licence, unless indicated otherwise in a credit line to the material. If material is not included in the article's Creative Commons licence and your intended use is not permitted by statutory regulation or exceeds the permitted use, you will need to obtain permission directly from the copyright holder. To view a copy of this licence, visit http://creativecommons.org/licenses/by/4.0/. The Creative Commons Public Domain Dedication waiver (http://creativeco mmons.org/publicdomain/zero/1.0/) applies to the data made available in this article, unless otherwise stated in a credit line to the data. 
injuries, particularly in the setting of an IOFB or soil contamination [11-14]. However, the causative microorganisms of posttraumatic endophthalmitis with and without retained IOFBs are less well known.

Our previous study reported that the locations and types of IOFBs were related to the development of endophthalmitis [15]. Therefore, it is necessary to determine whether the presence of intraocular foreign bodies in patients with traumatic endophthalmitis has any effect on the distribution of pathogenic microorganisms in endophthalmitis. Thus, the purposes of the current study were to review the demographic characteristics of patients with clinically diagnosed endophthalmitis and to analyze the causative microorganisms with or without IOFBs. These findings will provide a reference for guiding the treatment of endophthalmitis.

\section{Methods}

\section{Population}

A retrospective review was conducted of patients clinically diagnosed with endophthalmitis with open globe injuries at the Zhongshan Ophthalmic Center (ZOC), Sun Yat-sen University (Guangzhou, China) from January 1, 2013, to August 31, 2020. This study was performed in compliance with the principles of the Declaration of Helsinki and was approved by the Institutional Ethics Committee of Zhongshan Ophthalmic Center, Sun Yatsen University. In our study, clinical endophthalmitis was defined as any deterioration in clinical signs, such as hypopyon, decline in visual acuity, purulent discharge and positive microbial culture. The data in the case report format revealed no linkage to the patient identities, and the patient data confidentiality was protected. Data on demographic characteristics, including patient age and sex, medical records and laboratory results, were collected and analyzed. The type of IOFB was recorded as the name of the pathogenic object and classified as metallic or nonmetallic. The longest diameter of each IOFB was used to categorize the IOFBs into 4 size groups: $<3$ $\mathrm{mm}, 3-5 \mathrm{~mm},>5-10 \mathrm{~mm}$, and $>10 \mathrm{~mm}$.

\section{Pathogen isolation and identification}

Pathogen cultures were undertaken using patients' aqueous humor or vitreous or eye contents. The aqueous humor was aspirated from the anterior chamber through the limbus with a needle using a 1-mL syringe. Vitreous specimens were collected through the pars plana. Eye contents removed during enucleation were collected. The specimens were inoculated in nutrient broth overnight at $37^{\circ} \mathrm{C}$. Subsequently, the broth was inoculated onto sheep blood agar and potato glucose agar for the growth of bacterial and fungal cultures, respectively. Bacterial isolates were identified using an automated system (VITEK 2 compact BioMérieux, Inc., Marcy l'Étoile, France). Fungal isolates were identified by experienced technicians according to fungal morphology.

\section{Statistical analysis}

All analyses were performed using SPSS version 16.0 (SPSS Inc., Chicago, IL, USA). The characteristics of the study population and the culture results were summarized using means and standard deviations for continuous variables and percentages for categorical variables. Differences were considered to be significant at $P<0.05$.

\section{Results}

In the current study, a total of 1257 patients were clinically diagnosed with endophthalmitis with open globe injuries; among them, 1033 patients were cultured for bacteria and fungi, and 383 patients yielded positive cultures, with a positive culture rate of $37.1 \%$ (383/1033). The positive culture rate for the patients with IOFBs was $38.0 \%(146 / 384)$, while that in the patients without IOFBs was $36.5 \%(237 / 649)$. There was no significant difference between these two groups $(P=0.629)$. Of the 1257 patients with clinically diagnosed posttraumatic endophthalmitis, 452 (36.0\%) patients had IOFBs; furthermore, of the 383 patients with culture-proven endophthalmitis, 146 (38.1\%) had IOFBs. Of 452 patients with IOFBs, 376 had IOFBs removed in our hospital, including 6 patients with multiple foreign bodies, 3 with eyelashes and metallic bodies, and 3 with eyelashes and nonmetallic bodies. Therefore, a total of 382 IOFBs were removed, with $70.9 \%$ being metallic and $29.1 \%$ being nonmetallic bodies. The percentages of IOFBs within the $<3 \mathrm{~mm}, 3-5 \mathrm{~mm},>5-10$ $\mathrm{mm}$, and $>10 \mathrm{~mm}$ groups were $32.7,34.0,19.1$, and $14.1 \%$, respectively.

As shown in Table 1, of the 1257 clinically diagnosed endophthalmitis patients, 1051 patients were male, and 206 were female. In addition, 452 patients had IOFBs, and 805 patients did not have IOFBs. The proportion of males was $89.8 \%$ among patients with IOFBs, which was significantly higher than the $80.1 \%$ proportion of males among patients without IOFBs $(P<0.001)$. The age of the patients with clinically diagnosed endophthalmitis with IOFBs ranged from 1 to 78 years, with a mean of $38.3 \pm 15.6$ years, which surpassed the age of the patients without IOFBs, which ranged from 7 months to 78 years, with a mean of $27.9 \pm 20.5(P<0.001)$. Of the 383 patients with culture-proven endophthalmitis, the age of the patients with IOFBs ranged from 1 to 67 years, with a mean of $38.8 \pm 15.7$ years, and the age of the patients without IOFBs ranged from 8 months to 78 years, with a mean of $31.7 \pm 20.8$ years. The average age of the patients with IOFBs was older than that of the patients without IOFBs $(P<0.001)$. The proportions of males were $86.3 \%$ 
Table 1 Comparison of the demographic information of endophthalmitis with or without intraocular foreign bodies

\begin{tabular}{|c|c|c|c|c|c|c|}
\hline & \multicolumn{2}{|c|}{$\begin{array}{l}\text { Clinically diagnosed endophthalmitis } \\
(n=1257)\end{array}$} & \multirow[t]{2}{*}{$P$ value } & \multicolumn{2}{|c|}{$\begin{array}{l}\text { Culture-proven endophthalmitis } \\
(n=383)\end{array}$} & \multirow[t]{2}{*}{$P$ value } \\
\hline & With IOFBs & Without IOFBs & & With IOFBs & Without IOFBs & \\
\hline Sex & & & $P<0.001$ & & & $P=0.21$ \\
\hline Male & 406 & 645 & & 126 & 193 & \\
\hline Female & 46 & 160 & & 20 & 44 & \\
\hline Age & $38.3 \pm 15.6$ & $27.9 \pm 20.5$ & $P<0.001$ & $38.8 \pm 15.7$ & $31.7 \pm 20.8$ & $P<0.001$ \\
\hline Total & 452 & 805 & & 146 & 237 & \\
\hline
\end{tabular}

among the patients with IOFBs and $81.4 \%$ among the patients without IOFBs $(P=0.021)$.

The age distributions of the 1257 patients with clinically diagnosed posttraumatic endophthalmitis are shown in Fig. 1. The distribution of children aged between 0 and 10 was the most concentrated, followed by middle-aged patients between 41 and 50 years old. In the range of 11-50 years old, the prevalence increased with age, and the number of patients older than 60 years of age decreased sharply. Although the number of patients with IOFBs in the 41-50 age group was slightly greater than that without IOFBs, the numbers of patients without IOFBs in the other age groups were greater than those with IOFBs.

The distribution of 407 isolates of 383 patients is shown in Table 2. Overall, bacteria were more predominant pathogens than fungi. In detail, the most common causative pathogens were Gram-positive cocci (45.0 and 52.7\% in the groups with IOFBs and without IOFBs, respectively), followed by Gram-negative bacilli (27.2 and 20.7\% in the groups with IOFBs and without IOFBs, respectively). Gram-positive bacilli were more common in the patients with IOFBs than in those without IOFBs (17.9 vs. 9.4\%), accounting for 12.6 and $5.5 \%$, respectively. In detail, Bacillus cereus was the most common gram-positive bacilli and accounted for $10.6 \%$ in the patients with IOFBs and $4.3 \%$ in the patients without IOFBs. Fungi were less abundant in the patients with IOFBs than in those without IOFBs (8.0 vs. 15.6\%). In detail, there were 4 (2.7\%) Aspergillus spp. isolated from the patients with IOFBs, and 14 (5.5\%) were isolated from the patients without IOFBs. The detailed distributions of other pathogens are detailed in Table 2.

There were 21 patients with mixed infections, and the majority of them had double bacterial infections. Among them, there were 5 cases with IOFBs and 16 cases without IOFBs. Simultaneous infection with three pathogens only occurred in patients without IOFBs. Detailed information on the 21 patients is shown in Table 3.

\section{Discussion}

Posttraumatic endophthalmitis remains an important complication of open globe injuries, and IOFBs are a risk factor for the development of endophthalmitis. In this study, our goal was to compare the demographic characteristics and causative pathogens of endophthalmitis

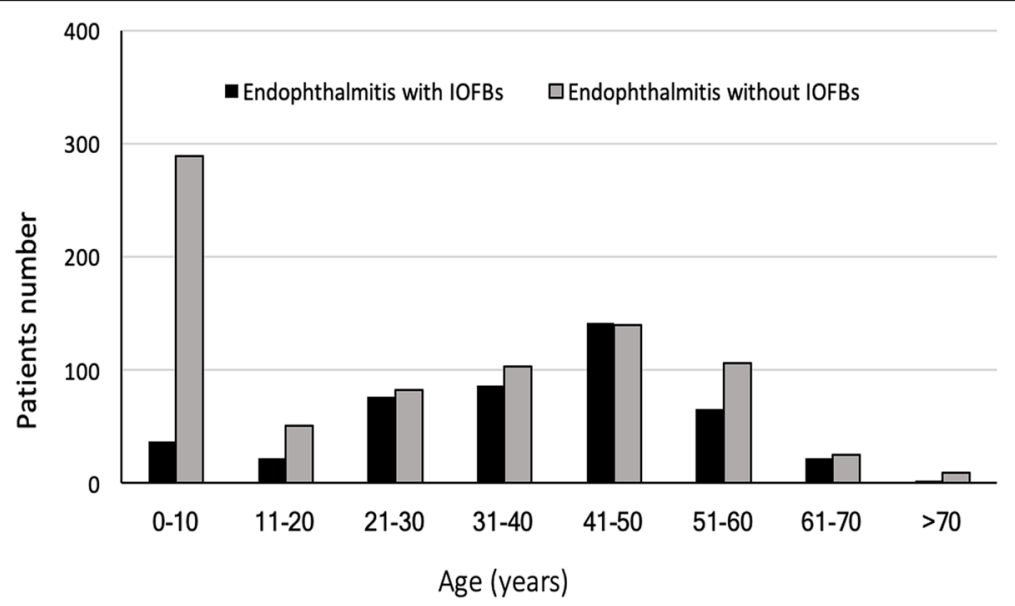

Fig. 1 Demographics of 1257 patients with clinically diagnosed posttraumatic endophthalmitis 
Table 2 Comparison of the causative isolates of endophthalmitis with or without intraocular foreign bodies

\begin{tabular}{|c|c|c|c|}
\hline & Endophthalmitis with IOFBs, $\mathrm{N}(\%)$ & $\begin{array}{l}\text { Endophthalmitis without IOFBs, } \mathrm{N} \\
\text { (\%) }\end{array}$ & $\begin{array}{l}\text { Total } \\
\mathrm{N}(\%)\end{array}$ \\
\hline Gram-positive cocci & $68(45.0)$ & $135(52.7)$ & $203(49.9)$ \\
\hline Coagulase-negative Staphylococcus & $52(34.4)$ & $74(28.9)$ & $126(31.0)$ \\
\hline Streptococcus & $7(4.6)$ & $32(12.5)$ & $39(9.6)$ \\
\hline Enterococcus & $4(2.7)$ & $9(3.5)$ & $13(3.2)$ \\
\hline Kocuria spp. & $1(0.7)$ & $8(3.1)$ & $9(2.2)$ \\
\hline Granulicatella & $3(2.0)$ & $1(0.4)$ & $4(1.0)$ \\
\hline Leuconostoc & - & $4(1.6)$ & $4(1.0)$ \\
\hline Staphylococcus aureus & - & $3(1.2)$ & $3(0.7)$ \\
\hline Others & $1(0.7)$ & $4(1.6)$ & $4(1.0)$ \\
\hline Gram-positive bacilli & $27(17.9)$ & $24(9.4)$ & $51(12.5)$ \\
\hline Bacillus cereus & $16(10.6)$ & $11(4.3)$ & $27(6.6)$ \\
\hline Bacillus subtilis & $3(2.0)$ & $3(1.2)$ & $6(1.5)$ \\
\hline Propionibacterium acnes & $1(0.7)$ & $3(1.2)$ & $4(1.0)$ \\
\hline Others & $7(4.6)$ & $7(2.7)$ & $14(3.4)$ \\
\hline Gram-negative cocci & $2(1.3)$ & $2(0.8)$ & $5(1.2)$ \\
\hline Neisseria & $2(1.3)$ & $2(0.8)$ & $4(1.0)$ \\
\hline Gram-negative bacilli & $41(27.2)$ & $53(20.7)$ & $94(23.1)$ \\
\hline Pseudomonas aeruginosa & $6(4.0)$ & $6(2.3)$ & $12(2.9)$ \\
\hline Enterobacter cloacae & $3(2.0)$ & $7(2.7)$ & $10(2.5)$ \\
\hline Aeromonas & $4(2.7)$ & $4(1.6)$ & $8(2.0)$ \\
\hline Klebsiella & $1(0.7)$ & $1(0.4)$ & $2(0.5)$ \\
\hline Acinetobacter & $5(3.3)$ & $1(0.4)$ & $6(1.5)$ \\
\hline Xanthomonas & $2(1.3)$ & $4(1.6)$ & $6(1.5)$ \\
\hline Serratia & $2(1.3)$ & $3(1.2)$ & $5(1.2)$ \\
\hline Escherichia coli & $1(0.7)$ & $3(1.2)$ & $4(1.0)$ \\
\hline Enteric bacilli & $2(1.3)$ & $3(1.2)$ & $5(1.2)$ \\
\hline Sphingomonas & $3(2.0)$ & $1(0.4)$ & $4(1.0)$ \\
\hline Pantoea & $1(0.7)$ & $2(0.8)$ & $3(0.7)$ \\
\hline Citrobacter & $3(2.0)$ & - & $3(0.7)$ \\
\hline Others & $8(5.3)$ & $18(7.0)$ & $26(6.3)$ \\
\hline Fungus & $12(8.0)$ & $40(15.6)$ & $52(12.8)$ \\
\hline Aspergillus spp. & $4(2.7)$ & $14(5.5)$ & $18(4.4)$ \\
\hline Candida & $1(0.7)$ & $4(1.6)$ & $5(1.2)$ \\
\hline Fusarium spp. & $4(2.7)$ & $7(2.7)$ & $11(2.7)$ \\
\hline Mucor & - & $4(1.6)$ & $4(1.0)$ \\
\hline Penicillium & - & $1(0.4)$ & $1(0.2)$ \\
\hline Others & $3(2.0)$ & $10(3.9)$ & $13(3.2)$ \\
\hline Actinomycetes & $1(0.7)$ & $2(0.8)$ & $3(0.7)$ \\
\hline Total & $151(100)$ & $256(100)$ & $407(100)$ \\
\hline
\end{tabular}

with or without IOFBs. We found that of 1257 patients with clinically diagnosed posttraumatic endophthalmitis, 452 (36.0\%) patients had IOFBs. Males dominated these two groups, and males were more common among the patients with IOFBs than among the patients without IOFBs. The average age of the patients with IOFBs was older than that of the patients without IOFBs. The most common microbial pathogens in these two groups were
Gram-positive cocci and Gram-negative bacilli. Grampositive bacilli were more common in the patients with IOFBs than in those without IOFBs (17.9 vs. 9.4\%), and Bacillus spp. accounted for 12.6 and 5.5\%, respectively. Fungi were less abundant in the patients with IOFBs than in those without IOFBs (8.0 vs. $15.6 \%$ ).

In the current study, among the 1257 patients with clinically diagnosed endophthalmitis and the 383 with 
Table 3 Mixed infections of 21 patients with posttraumatic endophthalmitis

\begin{tabular}{|c|c|c|c|}
\hline Patients & IOFBs & Bacteria & Fungus \\
\hline 1 & Yes & Staphylococcus saprophyticus & Fusarium \\
\hline 2 & Yes & Enterobacter cloacae & Mucor \\
\hline 3 & Yes & Bacillus cereus/Pseudomonas aeruginosa & - \\
\hline 4 & Yes & Pseudomonas aeruginosa/Micrococcus luteus & - \\
\hline 5 & Yes & Streptococcus anginosus/Staphylococcus epidermidis & - \\
\hline 6 & No & Bacillus cereus/Kocuria roseus & - \\
\hline 7 & No & Bacillus megaterium/Streptococcus gordonii & - \\
\hline 8 & No & Pantoea agglomerans/Staphylococcus epidermidis & - \\
\hline 9 & No & Propionibacterium bullosum/Escherichia coli & - \\
\hline 10 & No & Enterobacter albopictus/Streptococcus salivarius & - \\
\hline 11 & No & Staphylococcus epidermidis/Aeromonas sobria & - \\
\hline 12 & No & Staphylococcus epidermidis/Staphylococcus saprophyticus & - \\
\hline 13 & No & Staphylococcus epidermidis/Staphylococcus saprophyticus & - \\
\hline 14 & No & Bacillus cereus & Aspergillus flavus \\
\hline 15 & No & Staphylococcus epidermidis/Staphylococcus aureus & - \\
\hline 16 & No & Bacillus cereus/Abiotrophia defectiva & - \\
\hline 17 & No & Enterococcus gallinarum/Enterobacter albopictus & - \\
\hline 18 & No & Chryseobacterium indologenes/Delftia acidovorans & - \\
\hline 19 & No & Brevundimonas diminuta/Staphylococcus epidermidis & Aspergillus flavus \\
\hline 20 & No & Staphylococcus epidermidis/Streptococcus mutans/streptococcus oralis & - \\
\hline 21 & No & Staphylococcus epidermidis/Staphylococcus aureus/Mycobacterium & - \\
\hline
\end{tabular}

culture-positive endophthalmitis, the prevalence rates of IOFBs were 36.0 and $38.1 \%$, respectively, which were slightly lower than the $43 \%$ reported in a study of $67 \mathrm{cul}-$ ture-positive posttraumatic endophthalmitis patients [9]. Additionally, we found that endophthalmitis occurred predominantly in men $(83.6 \%)$, and the mean age of all the patients was $38.3 \pm 15.6$ years old. The mean age of the patients $(38.3 \pm 15.6)$ with IOFBs was significantly older than that of the patients $(27.9 \pm 20.5)$ without IOFBs. This finding might be due to sex-based behavior and male involvement in higher-risk working activities. In addition, only in the 41- to 50-year-old group were the patients close in number. In other age groups, the number of people without IOFBs was greater than that with IOFBs, especially in the age group under 10 years old. The great difference in the distribution of children under 10 years old between the two groups might explain why the average age of the patients with IOFBs was older than the age of those without IOFBs. A previous study in Southwest China reported that working-age males accounted for $79.1 \%$ of patients with IOFBs, and there were significant differences in age distributions [16], which is consistent with our results. Most previous studies of IOFBs have revealed that males predominate, and the average ages have been close to those in other findings. For example, Ratanapakorn et al. reported that males accounted for $93.3 \%$ of patients and that the mean age was 36.4 years for 359 consecutive patients with retained IOFBs in Thailand [17]. Anguita et al. reported that among 61 Latin American patients with IOFBs, males accounted for 97\%, and the mean age was 37.9 years old [18]. In England, Bourke et al. reported that 23 patients with IOFBs were all male, with a mean age of 37.4 years old [19].

The isolation of microbial pathogens is an important step in clinical practice. In the current study, Gram-positive cocci were found in 203 eyes (49.9\%), Gram-negative bacilli were found in 94 eyes (23.1\%), and Gram-positive bacilli were found in 51 eyes (12.5\%). Coagulase-negative Staphylococcus was the major causative pathogen, which is consistent with previous studies $[9,11-14,20]$. Gram-positive bacilli were more common in the patients with IOFBs than in those without IOFBs (17.9 vs. 9.4\%). Bacillus spp. is a group of uncommon but aggressive pathogens and accounts for approximately $1.5-10.7 \%$ of infectious endophthalmitis cases [11-14]. Furthermore, Bacillus spp. was more common in cases of endophthalmitis following open globe injuries than in cases of postoperative and endogenous endophthalmitis [21]. For example, Jindal reported that Bacillus spp. accounted for $17.1 \%$ of posttraumatic endophthalmitis cases [22], which was similar to our results. In addition, we found that Bacillus spp. were more common in the patients with IOFBs than in those without IOFBs (12.6 vs. 5.5\%). It has been reported that patients with traumatic ocular 
injuries have a high probability of being infected with Bacillus cereus if the injury is associated with a retained IOFB [23], which is consistent with our results. Fungal endophthalmitis following open-globe injuries is less common than bacterial endophthalmitis. In our series, fungi accounted for 8.0 and $15.6 \%$ in the patients with IOFBs and without IOFBs, respectively. The reasons for this difference still need to be further explored. We speculated that this might be related to the causes of ocular trauma and types of IOFBs. It has been reported that the most common agents in previous studies were Aspergillus, Candida and Fusarium [13, 14, 24, 25], which is consistent with our results. After comparison, we found that the distributions of most pathogens containing intraocular foreign bodies were similar, but there were different distributions of Bacillus species and fungi. In the current study, multiple infections accounted for 5.5\% (21/383) of cases, with $1.3 \%(5 / 383)$ detected in the patients with IOFBs and $4.2 \%(16 / 383)$ in the patients without IOFBs. A previous study of 1593 patients with infectious endophthalmitis $(82.6 \%$ with posttraumatic endophthalmitis) reported that $5.3 \%$ were infected by multiple pathogens [26], which was similar to our results.

The limitations of this study included its retrospective nature and the lack of visual acuity prognosis. Nevertheless, our study provides valid data for understanding the demographic characteristics and isolation of causative organisms from patients with or without IOFBs.

\section{Conclusions}

In this study, our results demonstrated that $36.0 \%$ of patients had IOFBs among cases of posttraumatic endophthalmitis. Male dominance was more common among patients with IOFBs than among patients without IOFBs. The average age of the patients with IOFBs exceeded that of the patients without IOFBs. The most common microbial pathogens were Gram-positive cocci and Gram-negative bacilli in these two groups. Grampositive bacilli were more common in the patients with IOFBs than in those without IOFBs. Fungi were less abundant in the patients with IOFBs than in those without IOFBs. Our findings demonstrated the different distributions of age, sex and microbial pathogens between patients with IOFBs and those without IOFBs.

\footnotetext{
Authors' contributions

YY and FM acquired the data and wrote the main manuscript text, JQL and $J \mathrm{YL}$ analyzed and interpreted the data, and KLW and FD conceived of and designed the study. All authors reviewed the manuscript. YY and FM contributed equally to this work. The author(s) read and approved the final manuscript.
}

\section{Funding}

This work was supported by funds from the Natural Science Foundation of Guangdong Province (2020A1515011364), National Natural Science
Foundation of China (81770896 and 81970848), and High-level Hospital Construction Project (303010401).

\section{Availability of data and materials}

All the data used to support the findings of this study are included within the article and are available from the corresponding author upon reasonable request.

\section{Ethics approval and consent to participate}

This study was performed in compliance with the principles of the Declaration of Helsinki and was approved by the Institutional Ethics Committee of Zhongshan Ophthalmic Center, Sun Yat-sen University. The Ethical Committee of Zhongshan Ophthalmic Center, Sun Yat-sen University waived the patients' consent given the retrospective nature of the study.Consent for publication

Not applicable.

\section{Competing interests}

The authors declare that they have no competing interests.

Received: 23 April 2021 Accepted: 24 September 2021

Published online: 25 October 2021

\section{References}

1. Loporchio D, Mukkamala L, Gorukanti K, et al. Intraocular foreign bodies: a review. Surv Ophthalmol. 2016;61:582-96.

2. Dehghani AR, Rezaei L, Salam H, Mohammadi Z, Mahboubi M. Post traumatic endophthalmitis: incidence and risk factors. Global J Health Sci. 2014;6:68-72.

3. Andreoli CM, Andreoli MT, Kloek CE, et al. Low rate of endophthalmitis in a large series of open globe injuries. Am J Ophthalmol. 2009;147:601-8 e2.

4. Essex RW, Yi Q, Charles PG, Allen PJ. Post-traumatic endophthalmitis. Ophthalmology. 2004;111:2015-22.

5. Zhang Y, Zhang MN, Jiang CH, Yao Y, Zhang K. Endophthalmitis following open globe injury. Br J Ophthalmol. 2010;94:111-4.

6. Verbraeken H, Rysselaere M. Post-traumatic endophthalmitis. Eur J Ophthalmol. 1994;4:1-5.

7. Williams DF, Mieler WF, Abrams GW, Lewis H. Results and prognostic factors in penetrating ocular injuries with retained intraocular foreign bodies. Ophthalmology. 1988;95:911-6.

8. Boldt HC, Pulido JS, Blodi CF, Folk JC, Weingeist TA. Rural endophthalmitis. Ophthalmology. 1989:96:1722-6.

9. Chhabra S, Kunimoto DY, Kazi L, et al. Endophthalmitis after open globe injury: microbiologic spectrum and susceptibilities of isolates. Am J Ophthalmol. 2006;142:852-4.

10. Teweldemedhin M, Gebreyesus H, Atsbaha AH, Asgedom SW, Saravanan M. Bacterial profile of ocular infections: a systematic review. BMC Ophthalmol. 2017;17:212.

11. Lin L, Mei F, Liao J, et al. Nine-year analysis of isolated pathogens and antibiotic susceptibilities of infectious endophthalmitis from a large referral eye center in Southern China. Infect Drug Resist. 2020;13:493-500.

12. Moloney TP, Park J. Microbiological isolates and antibiotic sensitivities in culture-proven endophthalmitis: a 15-year review. Br J Ophthalmol. 2014;98:1492-7.

13. Gentile RC, Shukla S, Shah M, et al. Microbiological spectrum and antibiotic sensitivity in endophthalmitis: a 25-year review. Ophthalmology. 2014:121:1634-42.

14. Liu C, Ji J, Li S, et al. Microbiological isolates and antibiotic susceptibilities: a 10-year review of culture-proven endophthalmitis cases. Curr Eye Res. 2017:42:443-7.

15. Duan F, Yuan Z, Liao J, et al. Incidence and risk factors of intraocular foreign body-related endophthalmitis in Southern China. J Ophthalmol. 2018;2018:8959108.

16. Chang T, Zhang Y, Liu L, et al. Epidemiology, clinical characteristics and visual outcomes of patients with intraocular foreign bodies in Southwest China: A 10-year review. Ophthalmic Res. 2021;64:494-502. 
17. Ratanapakorn T, Kongmalai P, Sinawat $S$, et al. Predictors for visual outcomes in eye injuries with intraocular foreign body. Clin Ophthalmol. 2020;14:4587-93.

18. Anguita R, Moya R, Saez V, et al. Clinical presentations and surgical outcomes of intraocular foreign body presenting to an ocular trauma unit. Graefes Arch Clin Exp Ophthalmol. 2021;259:263-8.

19. Bourke L, Bourke E, Cullinane A, O'Connell E, Idrees Z. Clinical outcomes and epidemiology of intraocular foreign body injuries in Cork University Hospital, Ireland: an 11-year review. Ir J Med Sci. 2021;190:1225-30.

20. Al-Omran AM, Abboud EB, Abu El-Asrar AM. Microbiologic spectrum and visual outcome of posttraumatic endophthalmitis. Retina. 2007;27:236-42.

21. Bhagat N, Nagori S, Zarbin M. Post-traumatic infectious endophthalmitis. Surv Ophthalmol. 2011;56:214-51.

22. Jindal A, Pathengay A, Mithal K, et al. Endophthalmitis after open globe injuries: changes in microbiological spectrum and isolate susceptibility patterns over 14 years. J Ophthal Inflamm Infect. 2014;4:5.
23. Mursalin MH, Livingston ET, Callegan MC. The cereus matter of Bacillus endophthalmitis. Exp Eye Res. 2020;193:107959.

24. Gupta A, Srinivasan R, Kaliaperumal S, Saha I. Post-traumatic fungal endophthalmitis--a prospective study. Eye. 2008;22:13-7.

25. Wykoff CC, Flynn HW Jr, Miller D, Scott IU, Alfonso EC. Exogenous fungal endophthalmitis: microbiology and clinical outcomes. Ophthalmology. 2008;115:1501-7 7.e1-2.

26. Yang XB, Liu YY, Huang ZX, et al. Clinical analysis of 1593 patients with infectious endophthalmitis: a 12-year study at a tertiary referral center in Western China. Chin Med J. 2018;131:1658-65.

\section{Publisher's Note}

Springer Nature remains neutral with regard to jurisdictional claims in published maps and institutional affiliations.
Ready to submit your research? Choose BMC and benefit from:

- fast, convenient online submission

- thorough peer review by experienced researchers in your field

- rapid publication on acceptance

- support for research data, including large and complex data types

- gold Open Access which fosters wider collaboration and increased citations

- maximum visibility for your research: over $100 \mathrm{M}$ website views per year

At BMC, research is always in progress.

Learn more biomedcentral.com/submissions 